\title{
ENTRE EL MERCADO Y LA CONSTRUCCIÓN LOCAL: REFLEXIONES PARA UNA GESTIÓN MÁS INCLUYENTE DE LA BIODIVERSIDAD Y LOS SERVICIOS ECOSISTÉMICOS EN EL MARCO DE LOS PAGOS POR SERVICIOS AMBIENTALES
}

\author{
Alexander Rincón Ruiz* \\ CÉSAR Rojas** \\ Margarita NiETO***
}

\section{Resumen}

Los esquemas de pagos por servicios ambientales (PSA) se han ratificado como mecanismo unidireccional preferido en la toma de decisiones para la gestión ambiental, particularmente en lo asociado a la gestión de la biodiversidad; adicionalmente, en el marco del reciente Decreto 0870 su relevancia a futuro de seguro será cada vez mayor. Esto se da en un contexto local donde las tendencias de gobernanza, con principios de participación e inclusión, enfocadas en procesos de construcción local y colectiva están en expansión. Este artículo avanza en el análisis del uso inercial de los PSA por la institucionalidad formal ambiental en Colombia. El análisis planteado propone lo oportuno de incluir los contextos territoriales de manera armónica en la adecuación e implementación de los instrumentos de

* PhD Ciencias Ambientales - Economía Ecológica. Profesor, Escuela de Economía, Universidad Nacional de Colombia, Bogotá (Colombia). [alrinconru@unal.edu.co].

** MSc Economía Ambiental. Consultor independiente. Bogotá (Colombia). [carojasj@unal.edu.co].

*** MSc Ciencias Ambientales - Economía Ecológica. Profesional Misional Organización de las Naciones Unidas para la Alimentación y la Agricultura (FAO). Bogotá (Colombia). [margaritamnieto@gmail.com].

Recibido: 24 de mayo de 2017 / Modificado: 28 de septiembre de 2017 / Aceptado: 19 de enero de 2018.

Para citar este artículo

Rincón Ruiz, A., Rojas, C. y Nieto, M. (2018). Entre el mercado y la construcción local: reflexiones para una gestión más incluyente de la biodiversidad y los servicios ecosistémicos en el marco de los pagos por servicios ambientales. OPERA, 22, pp. 103-118.

DoI: https://doi.org/10.18601/16578651.n22.06 
política ambiental, para consolidar una gobernanza orientada más al sentido de inclusión y creación de procesos de largo plazo. Por ello, la construcción de respuestas de política ambiental, particularmente los PSA en Colombia, deben darse a partir de cuatro puntos que desarrollamos como las 4C: conceptualización (qué y para qué), complementariedad (cómo), contexto (dónde) y construcción colectiva (con quién y para quién).

Palabras clave: biodiversidad, servicios ecosistémicos, política ambiental, pago por servicios ambientales, gobernanza ambiental, construcción colectiva.

\section{BETWEEN MARKET AND LOCAL PARTICIPATION: REFLECTIONS FOR BIODIVERSITY AND ECOSYSTEM MANAGEMENT IN THE CONTEXT OF PAYMENTS FOR ENVIRONMENTAL SERVICES}

\section{Abstract}

Payments for environmental services (PES) have been ratified as the preferred, unidirectional mechanism in decision-making for biodiversity management in Colombia, now regulated with Decree 0870. This happens in a local context where principles of participation and inclusion play as a major governance trend. This article discusses inertial use of PES in the current formal environmental Colombian institutions. It is proposed to harmonically include territorial contexts in PES adaptation and implementation, in order to consolidate inclusive and long term biodiversity governance. This, through a four points approach
(4C): conceptualization (what and what for), Complementarity (how), Context (where) and collective construction (with whom and for whom).

Key words: Biodiversity, ecosystem services, environmental policy, payment for environmental services, environmental governance, collective construction.

\section{INTRODUCCIÓN}

La transformación y la pérdida de la biodiversidad (BD) y los servicios ecosistémicos (SE) implican respuestas desde la política ambiental de un país, que para el caso de Colombia se ha reflejado en un avance en la definición de diferentes instrumentos de política para la gestión de la biodiversidad, por ejemplo, tasas por el uso de recursos, incentivos, tarifas, entre otros (Rodríguez-Becerra, Uribe y Carrizosa, 1996; Ecoversa, 2009; Rodríguez y Ávila, 2014).

No obstante, persiste la pérdida y transformación de la BD y los SE. Se identifica que el rol de la institucionalidad formal ambiental colombiana se ha caracterizado principalmente por estructuras instrumentales normativas y económicas que muchas veces no son acordes con las realidades de transformación del territorio, ni con una incorporación y armonización de las particularidades culturales de estos, por ejemplo, la restricción de usos del suelo que han sido tradicionales e históricos, en cumplimiento de normas de restricción completa de uso bajo un esquema de preservación; la formulación de planes, políticas y programas sectoriales sin el suficiente conocimiento y diferenciación de los contextos territoriales; el diseño de instrumentos sin el adecuado con- 
senso de los agentes que intervienen en su uso y aprovechamiento, entre otros (Hernández, Cárdenas, Mansilla y Baptiste, 1998; Hernández-Pérez, 2000, Ecoversa, 2009, Rodríguez y Ávila, 2014) y políticas homogéneas que no tienen en cuenta la heterogénea realidad local, como por ejemplo la política antidrogas (Rincón y Kallis, 2013)

Del mismo modo, los instrumentos de política ambiental, como los de control y seguimiento, por ejemplo, las licencias ambientales, restringen su aplicación a impactos ambientales y no incorporan la funcionalidad ecológica del territorio (Rincón, Toro y Burgos, 2009). Por su parte, los instrumentos económicos, como los mecanismos de pago por servicios ambientales (PSA), se han venido promoviendo de forma reiterativa en proyectos enfocados a la gestión de la biodiversidad, con varias lecciones aprendidas y aspectos por mejorar. En este artículo se hace una reflexión sobre los alcances de los PSA en el contexto de estas visiones entre la gobernanza participativa y la gobernanza de mercado, con énfasis en las múltiples oportunidades que abre la recién generada normatividad por la cual se establece el pago por servicios ambientales y otros incentivos a la conservación (Decreto 0870 del 25 de mayo del 2017), y la propuesta del documento de discusión "Lineamientos de política y programa nacional de pago por servicios ambientales para la construcción de paz" (Conpes 3886). Estos insumos conceptuales y operativos avanzan sobre la experiencia de problemáticas identificadas previamente en casos de formulación de PSA, y presentan grandes retos para su oportuna implementación en los territorios del país, con la adecuada participación de las comunidades locales.
El artículo se desarrolla en tres segmentos: primero, se expondrán las tendencias de la gobernanza ambiental y los instrumentos de política, presentando la dicotomía entre la gobernanza basada en el mercado y la gobernanza participativa. Posteriormente, se plantean reflexiones y recomendaciones en el planteamiento de instrumentos de política, con énfasis en el tema de PSA. Finalmente, se exponen las posibilidades para la determinación de instrumentos de política ambiental en la gestión del territorio, y las reflexiones y los retos hacia el futuro, particularmente en el caso de los PSA.

\section{TENDENCIAS DE LA GOBERNANZA AMBIENTAL E INSTRUMENTOS DE POLÍTICA: ENTRE LA GOBERNANZA BASADA EN EL MERCADO Y LA GOBERNANZA PARTICIPATIVA}

Meuleman 2008, citando a Kettl (2002), define el Gobierno como la estructura y función de las instituciones públicas, y la gobernanza como la forma en que el Gobierno hace su trabajo. Si bien el concepto de gobernanza ha tomado acepciones diversas (Subirats, 2010), un punto fundamental es que esta implica el reconocimiento, la aceptación y la integración de la complejidad como un elemento intrínseco al proceso político e implica un sistema de gobierno basado en la participación de actores diversos en el marco de redes plurales (Subirats, 2010).

Rhodes (1997) distingue varios usos del término gobernanza, de donde retomaremos dos: a) la gobernabilidad como estado mínimo: el uso de mercados y cuasimercados para pro- 
veer servicios públicos; b) la gobernanza como redes autoorganizadas: las redes desarrollan sus propias políticas y modelan sus ambientes. Meuleman (2008) habla de dos tipos de gobernanza: de mercado (market governance) y por redes (network gobernance). La gobernanza de mercado, junto con las nuevas formas de control jerárquico, constituyó la nueva gestión pública en los últimos años.

En este contexto, el Banco Mundial lideró una visión tecnocrática bajo la necesidad de instituciones estatales eficaces, que lograran el desarrollo en un contexto global de mercados liberalizados (Demmers, Fernández y Hogenboom, 2004), en la cual no se visualiza un papel relevante del gobierno nacional, sino principalmente, de mecanismos basados en el mercado, como la privatización, las pautas de conducta corporativa autodiseñadas (como la Responsabilidad Social Empresarial) y los mecanismos voluntarios (sistemas de certificación y compensación) (De Castro, Hogenboom y Baud, 2015). Esta tendencia contrasta con los desarrollos de autores como Ostrom (1990), que presentan la acción colectiva de los sistemas de gobierno local como aspecto fundamental en la regulación del acceso y uso de los recursos naturales. Bajo este contexto, la gobernanza supone una nueva posición de los poderes públicos en los procesos de gobierno, la adopción de nuevas funciones y la utilización de nuevos instrumentos para gobernar (Subirats, 2010). Es decir, mientras el paradigma tradicional establece una clara separación entre las esferas pública y privada, la gobernanza apunta hacia un escenario en donde la frontera entre las dos esferas se difumina, las responsabilidades hacia lo colectivo se distribuyen entre múltiples actores, y el poder político se encuentra absolutamente disperso entre una gran diversidad de sujetos (Subirats, 2010).

En la década de 1990 surgió la gobernanza por redes, basada en la cooperación entre gobiernos y actores sociales (Mueleman, 2008). Más allá de un análisis blanco y negro entre un tipo u otro de gobernanza surge la idea de metagobernanza (Jessop, 2004), que consiste en la "gobernanza de los diferentes tipos de gobernanza”, es decir, más allá de elegir un tipo de gobernanza contempla la posibilidad de su complementariedad. En estos términos el debate de Meuleman resulta pertinente al definir la gobernanza como el conjunto de interacciones de los gobiernos, otros organismos públicos, el sector privado y la sociedad civil, con el objetivo de resolver los problemas sociales o crear oportunidades sociales. Incluye instituciones, instrumentos, procesos y relaciones entre actores.

De otro lado, los instrumentos de política ambiental que contribuyen a la gestión de la BD y los SE, generalmente, pueden clasificarse en: 1) los instrumentos de regulación directa, denominados de comando y control, los cuales están basados en la promulgación de normas, en la ecuación coerción-sanción y en la forma tradicional de hacer cumplir la ley; 2) los instrumentos económicos, los cuales no deben ser entendidos necesariamente como "comodificación de la naturaleza" o asociarse estrictamente a mercado (Hahn et al., 2015). Sin embargo, gran parte de su desarrollo se ha dirigido a entender el mercado como mecanismo para propiciar el cumplimiento de las metas ambientales; 3) los instrumentos administrativos, consistentes en el otorgamiento 
de licencias, permisos y demás modos de adquirir el derecho a usar los recursos naturales previstos en las diferentes legislaciones; 4) la educación, la investigación, la asistencia técnica y la información ambiental conforman una cuarta categoría (Rodríguez y Espinoza, 2002), finalmente, 5) estarían las iniciativas basadas en los arreglos institucionales participativos, muchas veces informales, por ejemplo, los instrumentos de conservación basados en la acción colectiva, que pueden estar implícitos en la definición de instrumentos relacionados, como la cogestión; aquí se destacan aquellos que tienen que ver con la posibilidad de vincular a las comunidades y ofrecen la alternativa de ser diseñados bajo el fundamento de una relación entre Estado y sociedad civil. Los instrumentos que buscan involucrar agentes y se basan en la acción voluntaria necesitan de apoyo en su divulgación, conocimiento y de un mayor desarrollo jurídico, como es el caso de las herramientas de conservación privada (Solano, 2001; Jiménez, 2003).

No obstante esta diversidad, los PSA han tenido un creciente uso en los últimos años, tanto, que se puede decir que han llegado a constituirse en uno de los más importantes referentes de política ambiental. El pragmatismo de este enfoque aborda los dilemas de equidad, desarrollo sostenible y conservación desde el interior de la estructura basada en el mercado (De Castro et al., 2015), lo cual interesa a sectores que no están muy a favor de cambios estructurales que implican una mayor inclusión y transformación institucional. En este orden de ideas se destacan los esquemas de compensación, por ejemplo, el de reducción de las emisiones derivadas de la deforestación y degradación de los bosques en los países en desarrollo (REDD), como mecanismos para reemplazar la regulación estatal, mejorar la imagen corporativa y, posiblemente, solucionar conflictos. Por último, también satisface en parte la agenda global ambiental (que incluye organismos internacionales y ONG) (De Castro et al., 2015), la cual ha pasado gradualmente hacia una agenda de esquemas de compensación e incentivos basados en el mercado y pagos por servicios ambientales en sus diversas formas (Hall, 2012). No obstante, en el marco de estos esquemas, la participación de las comunidades locales se supedita a los términos de ser beneficiarias de prestaciones compensatorias, según la decisión de otros actores legitimados.

Bajo las nuevas ideas de gobernanza, donde las responsabilidades hacia lo colectivo se distribuyen entre múltiples actores y el poder político se encuentra disperso entre una gran diversidad de sujetos, la idea de gobernanza pretende precisamente estimular la transformación de las capacidades de gobierno e invita a aceptar la interacción entre niveles de gobiernos y la presencia de una red de actores diversos (Subirats, 2010). En este sentido, en las últimas décadas ha surgido un modo de gobernanza participativa que contrarresta -e incluso puede complementar- esta visión de gobernanza basada en el mercado. La participación de las organizaciones de la sociedad civil basada en discursos de justicia social, equidad y reducción de la pobreza se convirtió en un elemento central de la gobernanza ambiental en América Latina en las últimas décadas (De Castro et al., 2015). Además, se ha venido fortaleciendo por un creciente auge de movimientos ambienta- 
les locales. Desde esta visión, en lugar de una gestión basada en comando y control, o en el mercado, la gobernanza participativa se basa en alianzas entre actores claves para establecer objetivos y para diseñar e implementar iniciativas. La gobernanza participativa oscila entre los modelos de cogestión, en que el Estado y las comunidades locales desarrollan un plan sostenible para los territorios tradicionales (De Castro et al., 2015), y arreglos más complejos que incluyen a múltiples partes interesadas e instituciones multiescalares, como la gobernanza ambiental. El reto de los gobiernos es trasladar su modo de hacer tradicional, basado en lógicas "de arriba hacia abajo", de matriz jerárquica y burocrática, a formas de gobierno en red, o de gobernanza (Subirats, 2010).

En este contexto, y dada la expansión de instrumentos como los PSA, vale la pena traer a consideración elementos fundamentales más allá de las tradicionales respuesta basadas en un solo tipo de gobernanza, y tener en cuenta algo que puede asociarse a la posibilidad de trabajar con diferentes formas de esta, como lo propone Jessop (2004) con su concepto de metagobernanza, y lo visibiliza Ostrom (2013) al plantear la diversidad institucional (figura 1). Esto, en función de lograr resultados más eficientes y ajustados a la compleja realidad local, en casos como el colombiano. Esto puede complementar las reflexiones que se han dado en la literatura respecto a la tendencia creciente de la utilización de instrumentos de mercado en los últimos años -especialmente, la orientación hacia los PSA-; estas reflexiones críticas giran en torno a sus impactos en la escala de valores de la población (Kosoy y Corbera, 2010), su rigor científico (Naeem et al., 2015), así como su capacidad de incidencia (Lapeyre y Pirard, 2013).

\section{REFLEXIONES Y RECOMENDACIONES EN EL PLANTEAMIENTO DE INSTRUMENTOS DE MERCADO: PAGOS POR SERVICIOS AMBIENTALES}

Una vez aclarada la importancia de acercarse a una metagobernanza, se recomienda que previo a la determinación de los posibles instrumentos de política ambiental (particularmente los PSA) se debe tener en cuenta que los instrumentos de política son posibilidades de respuesta, y estas serán lo más apropiadas y eficaces en la medida que obedezcan a un diagnóstico adecuado, que además considere la problemática específica que se va a resolver, e incluya aspectos como capital social, dinámicas relacionadas con el uso de la BD y los SE, conflictos socioambientales, entre otros. En general, se han determinado cuatro elementos clave para tener en cuenta: conceptualización, complementariedad, contexto, construcción colectiva (en adelante 4C).

\section{Conceptualización}

Uno de los primeros vacíos identificados cuando se hace referencia a instrumentos económicos, y particularmente a pagos por servicios ambientales, son los bajos niveles de conceptualización, muchas veces es difícil definir sobre que se está hablando cuando se menciona PSA, ¿'se habla de subsidios?, ¿de instrumentos de mercado? ¿de arreglos voluntarios?, ¿públicos o privados?, ¿pagos en dinero o especie? La literatura y la experiencia de otros países permite identificar múltiples posibilidades, por ejemplo, algunos casos emblemáticos como Costa Rica funcionan más como un subsidio 
que como un incentivo; en México se da un componente importante al tema de pobreza y no simplemente un pago por un "servicio ambiental”. De la misma forma, experiencias con pagos monetarios en algunas zonas de África llamarían la atención por preferir pagos en especie (Finlayson, 2013).

Como lo plantean Hahn et al. (2015), cuando se habla de instrumentos económicos se relaciona con "comodificación de la naturaleza” (valoración monetaria), sin embargo, podría no ser siempre así. De igual forma, cuando se señalan los esquemas de pagos por servicios ambientales se piensa en instrumentos de mercado (de hecho son clasificados como tales); no obstante, esto no sería así necesariamente si se tiene en cuenta que muchos de los PSA implementados obedecen más a esquemas tipo acuerdos voluntarios o subsidios (Muradian, Corbera, Pascual, Kosoy y May, 2010; Lapeyre y Pirard, 2013).

Los PSA se han constituido en Colombia como un instrumento de alto interés político para ser desarrollado por agencias y organizaciones para la conservación y el mantenimiento de servicios ambientales, principalmente los hídricos (Fondo Patrimonio Natural, 2014). En la taxonomía de instrumentos de política ambiental nacional, los PSA convergen en su diseño en arreglos institucionales entre diferentes agentes que implican el cumplimiento, según Wunder (2006), de los siguientes requisitos: 1) una transacción voluntaria; 2) un servicio ambiental bien definido (o un uso de la tierra que provea ese servicio); 3) un comprador; 4) un proveedor; y, 5) si y solo si, el proveedor asegura la provisión de ese servicio, determinado algunas veces como principio de condicionalidad.

En Borda, Moreno y Wunder (2010) se reconoce que los PSA pueden ser de dos tipos: puros y los llamados esquemas “tipo PSA". Así, cuando cumplen con los cinco criterios mencionados se define como un "PSA puro", en el sentido de que ofrecen compensaciones voluntarias directas y condicionadas a la provisión del servicio (Borda et al., 2010, p. 14). Por otro lado, cuando no se cumple con los criterios, por ejemplo, al no hacer las compensaciones directas a los proveedores de los servicios y no existir una condicionalidad clara en el pago, se consideran como esquemas "tipo PSA". Se puede decir que estos esquemas "tipo PSA" son los de mayor expansión en Colombia. De acuerdo con quién financia los PSA se puede hablar de PSA público - financiado por un organismo del Gobierno-y privado-financiado directamente por usuarios-.

Muradian et al. (2010) proponen una nueva definición de los PSA, como una transferencia de recursos entre los actores sociales, cuyo objetivo es crear incentivos para alinear las decisiones individuales de la tierra y el uso colectivo con el interés social en la gestión de los recursos naturales. Tales transferencias, que pueden ser monetarias o no monetarias, están determinadas por los valores y las percepciones que son determinantes en el diseño del acondicionamiento de los PSA y sus resultados (Muradian et al., 2010). En esta definición se aceptan no solo las transferencias a través de un mercado, sino también otros mecanismos, como los incentivos o subsidios públicos definidos por vía reglamentaria. De acuerdo con 
Muradian et al. (2010) y Tacconi (2012) existe una gran diversidad de iniciativas para los PSA que se agrupan de acuerdo con la importancia de los incentivos económicos, la inmediatez de la transferencia y el grado de mercantilización de los servicios ambientales.

Según Muradian et al. (2010) existen algunos aspectos críticos que deben tenerse en cuenta en el diseño y la formulación de los PSA con el fin de garantizar una adecuada asignación de los SE en el largo plazo, correspondientes a: 1) el alcance de la participación del Estado y la comunidad en las transacciones de mercado; 2) la definición de los SE y su relación causal con las prácticas de uso del suelo; 3) el cumplimiento de criterios de distribución social en su asignación, por ejemplo, relacionados con la solución de la pobreza y distribución de beneficios; y, 4) el rigor de la conservación y el pago.

En este sentido, las iniciativas de los PSA en América Latina, y en Colombia en particular, no cumplen con los cinco criterios de un PSA puro (Borda et al., 2010; Muradian et al., 2010; Tacconi, 2012). Varios análisis, similares al anterior, han dado lugar a plantear la necesidad de redefinir los PSA puros. Entre las razones que justifican esta reflexión se encuentran: su aplicación y ejecución limitadas, y que los supuestos detrás de la conceptualización teórica y metodológica no se cumplen por la naturaleza misma de los SE y el tipo de relaciones que se establecen entre ellos y las comunidades. Además, el soporte de información y conocimiento para la toma de decisiones es limitado (Naeem et al., 2015). Por ejemplo, existen casos en los que las comunidades no ven los SE como simples mercancías transables y establecen otro tipo de valoración al respecto. De igual manera, los SE son a menudo bienes de carácter público -locales y globales-y, por tanto, su disposición supone un problema de acción colectiva en su asignación (Cárdenas y Maldonado, 2013).

En este orden de ideas resulta fundamental, como paso inicial, un nivel de conceptualización apropiado para tener muy claro realmente qué se quiere en materia de instrumentos económicos y pagos por servicios ambientales. Su apropiado diseño depende de una adecuada conceptualización que permita identificar las diferentes posibilidades, más allá de un esquema homogéneo de incentivos monetarios. En este punto, el marco normativo de los PSA y los lineamientos de política expresados en el reciente Decreto 0870 y el Conpes 3886 de PSA tienen avances significativos que deben ser aprovechados, por ejemplo, contemplar diferentes posibilidades entre PSA públicos y privados, pagos en especie y en dinero, y avanzar sobre los llamados "PSA ampliados" expuestos por Muradian, más allá de una visión reduccionista de los llamados "PSA puros", pues muchos no cumplirían las condiciones estipuladas por Wunder.

\section{Contexto}

Dado el carácter de "respuesta formal" de los PSA y, en general, de los instrumentos de mercado, su diseño e implementación debe tener preguntas claras vinculadas a contextos territoriales. Uno de los retos es que este tipo de instrumentos no se convierta en algo de carácter exclusivamente técnico y al parecer "neutral", presentándose como respuestas anticipadas 
que no reflejan las complejidades territoriales y los vacíos institucionales existentes. De allí que resulte fundamental entender la configuración de los territorios y las circunstancias locales, para determinar su viabilidad, independientemente de aspectos como la disponibilidad de pagar. En determinados contextos es posible que los llamados instrumentos de mercado no sean la respuesta más acertada, y podrían convertirse en incentivos perversos frente a objetivos de gestión ambiental; para evitar esto es necesario analizar su complementariedad con otros instrumentos (factor que se expondrá más adelante), y discutir la viabilidad de tener incentivos no monetarios. En este campo, el Decreto 0870 y el Conpes 3886 de PSA reconocen la importancia de tener en cuenta los contextos locales, lo cual es un gran avance; sin embargo, no deja de ser un gran desafío, ya que en la práctica su inclusión depende de que se posibiliten acercamientos, estudios y canales adecuados para el entendimiento de los contextos territoriales a fin de tener un adecuado diagnóstico.

La identificación de los actores y la comprensión de sus relaciones en el territorio, sus alianzas, las asimetrías de poder entre ellos, el capital social existente, el reconocimiento de los diferentes valores ecológicos y sociales, y la existencia de conflictos ambientales, entre ellos los basados en trade offs entre servicios ecosistémicos, son fundamentales para entender la realidad territorial, y así definir y evaluar la viabilidad de la implementación de posibles instrumentos.

En contextos complejos y conflictivos, la pregunta clave para los actores involucrados en el proceso de gestión de la BD y los SE puede ser, ¿cuáles son los mejores instrumentos de política por utilizar para posibilitar procesos de construcción de gobernanza local? Para esto se debe partir de la premisa de que las decisiones finalmente no son técnicas sino políticas. En este orden de ideas, incentivos como los PSA, contextualizados a la realidad local pueden tener un potencial en la construcción de gobernanza local, si contribuyen a la construcción de procesos de inclusión.

\section{Complementariedad}

Otro aspecto fundamental de reflexión acerca de la expansión de esquemas como los PSA es lo comentado por autores como Ostrom y Walker (2003) sobre la importancia de tener en cuenta la "diversidad institucional" mencionada anteriormente, es decir, si bien el mercado es una institución que dentro de la economía tradicional se considera eficiente -bajo el paradigma de considerar la problemática ambiental como una falla de mercado que debe ser corregida, estos instrumentos se consideran apropiados-; no obstante, si se tiene en cuenta el contexto de complejidad y conflictividad socioambiental de Colombia, es posible que los instrumentos asociados al mercado no sean suficientes $y$, a veces, sean contraproducentes o simplemente necesitan ser complementados por instrumentos de largo plazo.

En contextos de interés para la gestión de la $\mathrm{BD}$ y los SE, donde la complejidad emerge desde las relaciones ecológicas y los distintos actores e intereses, la gestión implica tener en cuenta la diversidad institucional (figura 1) y utilizar instrumentos más allá del dominio exclusivo de los instrumentos de mercado y las 
estrategias de comando y control, que puedan complementarse con instrumentos de largo plazo, ejemplo de estos pueden ser los acuerdos informales, la construcción de capital social, las inversiones locales, entre otros (Rincón, 2014). En estos casos, trabajar en otros "niveles" de instituciones -formales e informales- es un paso esencial para una gestión del territorio de largo plazo (figura 1). Tener en cuenta un único nivel institucional -por ejemplo el mercado- podría no ser lo más adecuado y podría llevar al capital natural a su deterioro (Martín, Gómez y Montes, 2009). Esta afirmación se asocia a lo que Cárdenas (2009) describiría como dilemas de lo colectivo.
Esta diversidad institucional se relaciona con la importancia de diferentes tipos de instrumentos de tal forma que se complementen; se espera que así se puedan generar procesos de largo plazo y sostenibles, frente a las soluciones técnicas coyunturales que ofrecen respuestas de corto plazo y no comprometen cambios estructurales en contextos territoriales reales. Es posible que la viabilidad de instrumentos como los PSA se incremente complementando su creación con la consolidación de capital social, participación local, empoderamiento de las comunidades, entre otros. Este factor de la complementariedad está presente en el Decreto de PSA, pero la misión de llevarlo a la práctica será un reto clave para su éxito.

FIGURA 1. DIVERSIDAD INSTITUCIONAL Y GESTIÓN ASOCIADA

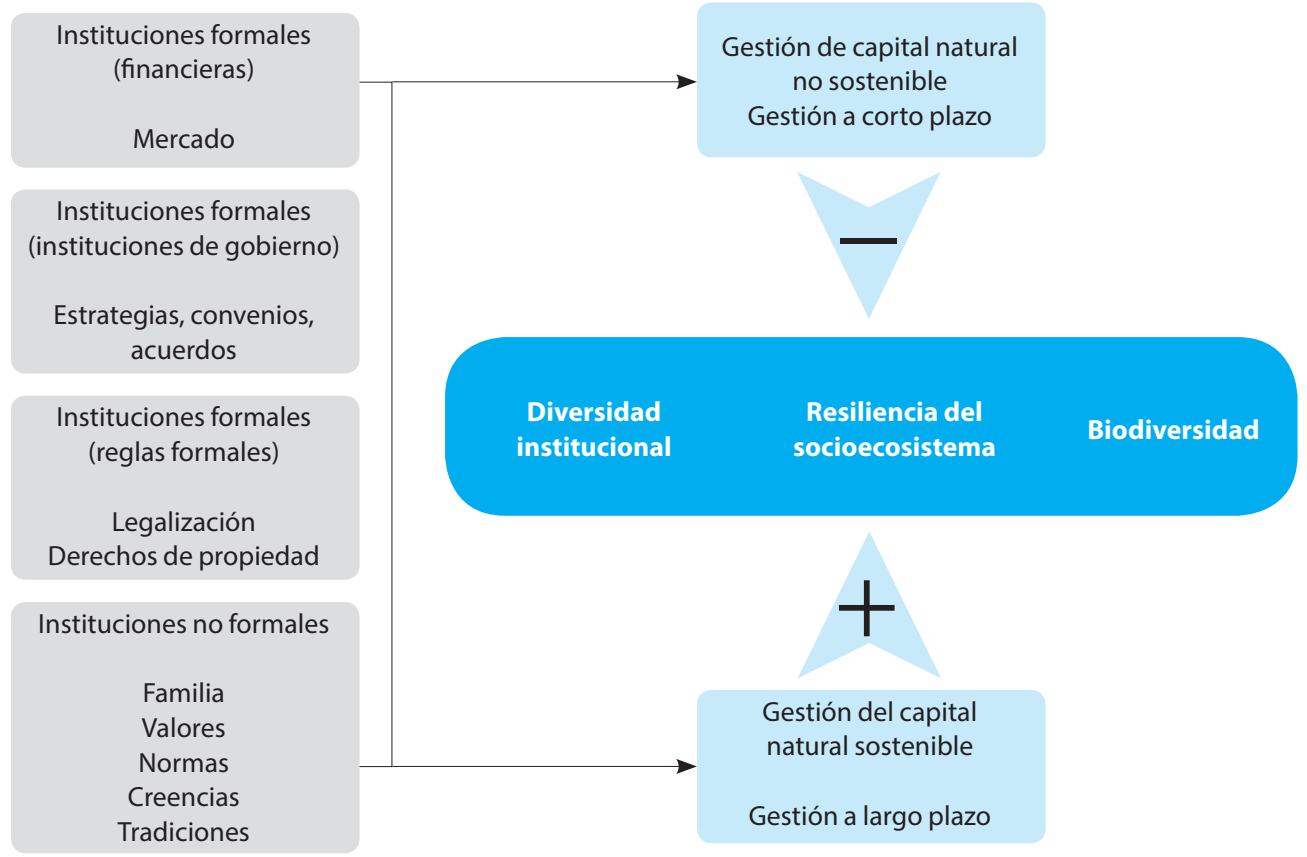

Fuente: Martín López et al. (2009). 


\section{Construcción colectiva}

Un escenario ideal en la negociación de acciones para la conservación de la biodiversidad en determinados territorios cuenta con tener la información de base necesaria - pertinente y suficiente- para construir el conocimiento. Sin embargo, no resulta tan viable y evidente garantizar que la disponibilidad de información sea suficiente para traducirla en conocimiento útil en la gestión del territorio. En este sentido, se requiere que tanto la construcción del conocimiento como la toma de decisiones se den en un escenario constructivo y permanente de concertación entre actores, es decir, configurar una gobernanza adaptativa que responda a las dinámicas territoriales (Cárdenas y Maldonado, 2013). De Groot, Alkemade, Braat, Hein y Willemen (2010) resaltan la importancia de tener elementos que deben ser considerados en forma previa en la determinación de instrumentos de política ambiental, particularmente, los asociados a las relaciones de oferta y demanda de los SE. La inclusión de actores se vislumbra en el Decreto 0870 y el Conpes 3886 de PSA. En el instrumento normativo se hace explícita la importancia de complementarse con procesos de comando y control, procesos asociativos y economías de escala para la conservación y mejora productiva. El resto está en hacer efectiva esta inclusión desde los mismos actores, cómo se piensa el territorio desde lo local y qué iniciativas se proponen para poder ser complementadas con esquemas tipo PSA. La existencia de intermediarios que desarrollan estos esquemas, pero no incluyen a las comunidades locales en estos procesos, opaca las posibilidades de incidencia, luego se convierte en un desafío grande.
Finalmente, con los elementos clave expuestos se proponen principios mínimos para la construcción de procesos de gestión de la $\mathrm{BD}$ y los SE de carácter incluyente, de largo plazo y enfocados hacia una integralidad territorial, en el marco de los esquemas tipo PSA en el contexto del Decreto 0870 y el Conpes 3886 de PSA.

\section{EL ANÁLISIS DE ESCENARIOS: UNA POSIBILIDAD PARA LA DETERMINACIÓN DE INSTRUMENTOS DE POLÍTICA AMBIENTAL PARA LA GESTIÓN DEL TERRITORIO}

Teniendo en cuenta los cuatro elementos planteados anteriormente (conceptualización, contexto, complementariedad y construcción colectiva), una de las herramientas que podría permitir incluir esta diversidad de elementos es el análisis de escenarios. La fase de construcción de escenarios bajo una buena base técnica y un proceso participativo es clave en el proceso de toma de decisiones y construcción de la gobernanza. Los escenarios son particularmente apropiados para evaluar el desarrollo de sistemas complejos (Raskin, Gallopin, Gutman, Hammond y Swart, 1998; Patel, Kok y Rothman, 2007), en pro del bienestar humano y la sostenibilidad. Peterson, Cumming y Carpenter (2003) consideran que los escenarios ofrecen un marco de trabajo sobre cómo, cuándo y dónde actuar para el desarrollo de políticas de conservación más flexibles y adaptativas, bajo panoramas de incertidumbre.

A su vez, en el análisis de escenarios pueden confluir tanto aspectos técnicos como participativos de concertación que, al final, son los que pueden llevar a determinar el conjunto de 
instrumentos más apropiados bajo el contexto y la problemática local inmediata. En este sentido, el grado de éxito de un instrumento de política particular depende de la interacción de diferentes factores relacionados como los mencionados por Rodríguez y Espinoza (2002), a saber: 1) la naturaleza de los problemas ambientales abordados; 2) los actores involucrados y sus visiones sobre la relación sociedad-ambiente; 3) las condiciones económicas, políticas y sociales; 4) el contexto legal; 5) las capacidades de gestión para implementar el instrumento; 6) las necesidades de coordinación y unión de voluntades para incorporar las distintas sensibilidades de los actores; 7) la integración con otros instrumentos.

\section{REFLEXIONES Y CONCLUSIONES}

Las recomendaciones expresadas en los planteamientos $4 \mathrm{C}$ pueden ayudar a que la implementación de esquemas basados en el mercado, como PSA, puedan ser llevados a cabo sin generar posibles conflictos futuros. En la tabla 1 se dan algunos contextos que permiten resumir la importancia de una visión más integral alrededor de instrumentos de mercado.

El marco normativo de PSA (decreto y Conpes) plantea en diferentes puntos su importancia en procesos como la disminución de cultivos de uso ilícito, lo cual se une a compromisos del acuerdo de paz. Sobre este punto ya hay un aprendizaje a partir de la política antidrogas y la lucha contra el narcotráfico, cuyos problemas asociados al no entendimiento de las realidades locales han sido descritos en la literatura (Rincón y Kallis, 2013; Rincón, Pascual y Flantua, 2013a), así como los retos a futuro asociados a un entendimiento de los contextos rurales más allá de una política de incentivos descontextualizada de las particularidades territoriales del país.

Bajo las anteriores premisas y reflexiones se tiene que para una acertada generación de instrumentos de política para la gestión de la BD y los SE en Colombia, la sostenibilidad financiera no debería ser el elemento fundamen-

TABLA 1. RECOMENDACIONES

\begin{tabular}{|l|l|l|}
\hline \multicolumn{1}{|c|}{ PSA (visión no integral) } & \multicolumn{1}{c|}{ Recomendaciones } \\
\hline Criterio & Gobernanza basada en el mercado & Gobernanza participativa, metagobernanza \\
\hline Enfoque & $\begin{array}{l}\text { Garantizar la provisión de un servicio } \\
\text { ecosistémico }\end{array}$ & Gestión integral del territorio \\
\hline Financiamiento & $\begin{array}{l}\text { Demandantes de los servicios eco- } \\
\text { sistémicos }\end{array}$ & $\begin{array}{l}\text { Actores asociados al servicio ecosistémico y búsqueda con- } \\
\text { junta con otras instituciones y actores }\end{array}$ \\
\hline Información & $\begin{array}{l}\text { Información para delimitar el pro- } \\
\text { blema ambiental }\end{array}$ & $\begin{array}{l}\text { Información necesaria para la comprensión integral del te- } \\
\text { rritorio para la toma de decisiones, acuerdos y negociación. }\end{array}$ \\
\hline Valoración & Valoración económica & Valoración integral \\
\hline
\end{tabular}

Fuente: elaboración propia. 
tal de reflexión, sino más bien la capacidad de los PSA para generar procesos locales de largo plazo, más allá de una continua dependencia de recursos financieros. Esta será la forma como los PSA se complementen con otros instrumentos, se adapten a los contextos locales y logren hacer partícipes a los diferentes actores en su diseño, a fin de entender realmente la sostenibilidad de los esquemas de PSA. Si el Decreto de PSA se desarrolla pensando sobre todo en la construcción de procesos de largo plazo a nivel local, cambios de sistemas de producción, generación de gobernanza local, puede convertirse en un instrumento fundamental en la construcción y gestión de la BD y SE; caso contrario si se convierte en el clásico incentivo monetario (gobernanza de mercado), muy enmarcado en la óptica de la economía ambiental y visión reduccionista de internalización de externalidades, sin ninguna visión de integralidad. El Decreto 0870 y el Conpes de PSA presentan la dicotomía entre una gobernanza basada en el mercado y la gobernanza local participativa. Sin lugar a dudas, la nueva normatividad tiene los elementos para hacer esto posible, el reto está ahora en que se tenga la voluntad política y técnica, pues existen visiones tradicionales muy centradas en el mercado que no dan paso a formas más integrales de gestión de BD y SE. La idea es superar estas visiones y hacer de la apuesta de PSA para el país una estrategia de consolidación de procesos sostenibles de largo plazo.

El diseño y la implementación de los instrumentos de política ambiental se han limitado a respuestas desde la institucionalidad formal ambiental. Las reflexiones acerca del diseño de nuevos instrumentos exponen la necesidad de reformar la visión sobre la participación social en la conservación como proceso emergente, así como la importancia de la inclusión de actores locales que participen en el desarrollo de nuevos instrumentos, sus responsabilidades e intereses en el territorio, la complementariedad con otros instrumentos existentes, el fortalecimiento de capacidades, la construcción de tejido social y la toma de decisiones informada.

En este sentido, se reconoce una ventaja en la construcción de procesos territoriales a partir del diagnóstico previo, en función de identificar los dilemas de uso y las sinergias entre los SE y los actores, la construcción de escenarios y la evidente necesidad de gestionarlos y desarrollarlos a partir de la articulación y armonización de instrumentos de carácter social y económico con acciones de conservación adaptadas al contexto territorial.

Los PSA se han constituido en Colombia en uno de los instrumentos más recomendados en materia ambiental y económica para la solución de temas asociados a la asignación de los SE. Los instrumentos deben ser evaluados por la forma en que estos son aplicados, más que por su definición misma; en el caso de los PSA las preguntas son: ¿las formas son las más adecuadas? ¿Se están entendiendo los contextos locales? ¿Se está teniendo en cuenta la diversidad institucional? ¿Su propuesta parte de un diagnóstico de la realidad territorial? ¿Se contempla la diversidad de trade offs existentes entre SE? ¿Se hacen pensando en un diagnóstico de la complejidad y conflictividad local? ¿Son una salida que tiene legitimidad social?, entre otras. 
En este sentido, el Decreto de PSA tiene el reto de hacer un enlace entre los clásicos incentivos de mercado y la construcción de una gobernanza local incluyente, que contemple las reflexiones planteadas en este texto. Tanto el marco normativo como los lineamientos de política del PSA contienen elementos para hacer esto posible. A pesar de que se debe pensar en la sostenibilidad financiera (el cuánto), un factor que no se debe olvidar es cómo se utilizarán; si son destinados a incentivar, continuar y construir procesos sostenibles de largo plazo, bajo los preceptos de contexto, conceptualización, complementariedad y construcción colectiva expuestos en este artículo, pueden tener un adecuado impacto en el territorio.

\section{AGRADECIMIENTOS}

Agradecimientos especiales al Instituto Alexander von Humboldt, donde inició la idea de realizar este artículo, y desde donde tuvimos todo el apoyo para su desarrollo durante el tiempo que estuvimos vinculados al Instituto.

\section{REFERENCIAS}

Borda, C. A., Moreno-Sánchez, R. P. y Wunder, S. (2010). Pagos por servicios ambientales en marcha: la experiencia en la microcuenca de Chaina. Departamento de Boyacá, Colombia. Brasil: Center for International Forestry Research (Cifor).

Cárdenas, J. C. (2009). Dilemas de lo colectivo: instituciones, pobreza y cooperación en el manejo local de los recursos de uso común. Bogotá: Ediciones Uniandes.
Cárdenas, J. C. y Maldonado, J. H. (2013). El papel de las comunidades locales en la conservación, el manejo de los recursos de uso común y sus políticas públicas. Notas de Política, 15. Bogotá: CEDE. Universidad de los Andes.

De Castro, F., Hogenboom, B. y Baud, M. (2015). Introducción. Gobernanza ambiental en América Latina en la encrucijada, moviéndose entre múltiples imágenes, interacciones e instituciones. En de Castro, F., Hogenboom, B. y Baud, M. (coords.). Gobernanza ambiental en América Latina. Buenos Aires: Clacso.

De Groot, R., Alkemade, R., Braat, L., Hein. L. y Willemen, L. (2010). Challenges in integrating the concept of ecosystem services and values in landscape planning, management and decision making. Ecological Complexity, 7, 260-272. doi: 10.1016/j.ecocom.2009.10.006

Demmers, J., Fernández, A. E. y Hogenboom, B. (eds.) (2004). Good Governance in the era of global neoliberalism: Conflict and depolitisation in Latin America, Eastern Europe, Asia, and Africa. New York: Routledge.

Departamento Nacional de Planeación (DNP) - Consejo Nacional de Política Económica y Social (Conpes), República de Colombia (2017). Documento Conpes 3886. Lineamientos de Politica y Programa Nacional de Pago por Servicios Ambientales para la Construcción de Paz. Bogotá: DNP-Conpes.

Ecoversa (2009). Recopilación, evaluación y análisis de los instrumentos económicos y tributarios existentes en materia ambiental. Informe Técnico, Ecoversa - Colombia.

Finlayson, R. (2013). Africa's ecosystem-services schemes face many challenges. World Agroforestry Center.

Fondo Patrimonio Natural (2014). Proyecto Incentivos a la conservación para el manejo del territorio y la 
mitigación de conflictos socio ambientales: resumen de logros y aprendizajes. Bogotá: FPN.

Hahn, T., Olsson, P., Folke, C. y Johansson, K. (2006). Trust-building, knowledge generation and organizational innovations: The role of a bridging organization for adaptive comanagement of a wetland landscape around Kristianstad, Sweden. Human Ecology, 34 (4), 573-592.

Hahn, T., McDermott, C., Ituarte-Lima, C., Schultz, M., Green, T. y Tuvendal, M. (2015). Purposes and degrees of commodification: Economic instruments for biodiversity and ecosystem services need not rely on markets or monetary valuation. Ecosystem Services. 16, 74-82.

Hall, A. (2012). Forests and Climate Change. The Social Dimensions of REDD in Latin America. Cheltenham / Northampton: Elgar Publishing.

Hernández, S., Cárdenas, J., Mansilla, H. y Baptiste, L. G. (1998). Valoración y diseño de politicas económicas para la gestión de la biodiversidad a nivel local. Bogotá: Instituto Alexander von Humboldt y Departamento Nacional de Planeación.

Hernández-Pérez, S. (ed.). (2000). Incentivos para la conservación y uso sostenible de la biodiversidad. Bogotá: Instituto de Investigación de Recursos Biológicos Alexander von Humboldt, DNP, wwF, RNSC, UAESPNN.

Jessop, B. (2004). Multi-level governance and multilevel meta-governance. En Bache, E. y Flanders, M. (eds.). Multi-level Governance (pp. 49-74). Oxford: Oxford University Press.

Jiménez, R. A. (2003). Marco conceptualy legal de las servidumbres ecológicas en Colombia y Latinoamérica. Santiago de Cali: Asociación Red Colombiana de Reservas Naturales de la Sociedad Civil.

Kosoy, N. y Corbera, E. (2010). Payments for ecosystem services as commodity fetishism. Ecological Economics, 69, 1228-1236.
Lapeyre, R. y Pirard, R. (2013). Payments for environmental services and market-based instruments: Next of kind or false friends? Working Paper. IDDRI. SciencesPo. Valuations, Markets and Policies for Biodiversity and Ecosystem Services. Martín-López, B., Gómez-Baggethun, E. y Montes, C. (2009). Un marco conceptual para la gestión de las interacciones naturaleza-sociedad en un mundo cambiante. Cuides, 3, 229-258.

Meuleman, L. (2008). Public Management and the Metagovernance of Hierarchies, Networks and Markets. The Feasibility of Designing and Managing Governance Style Combinations. Heidelberg: Physica-Verlag.

Ministerio de Ambiente y Desarrollo Sostenible (MADS) (2017). Decreto 0870. Recuperado de http:// es.presidencia.gov.co/normativa/normativa/ DECRETO\%20870\%20DEL\%2025\%20DE\%20 MAYO\%20DE\%202017.pdf

Muradian, R., Corbera, E., Pascual, U., Kosoy, N. y May, P. (2010). Reconciling theory and practice: An alternative conceptual framework for understanding payments for environmental services. Ecological Economics, 69, 1202-1208.

Naeem, S. et al. (2015). Get the science right when paying for nature's services. Science, 347 (6227), 1206-1207.

Ostrom (1990). Governing The Commons: The Evolution of Institutions for Collective Action. Cambridge, U.K.: Cambridge University Press.

Ostrom, E. (2013). Comprender la diversidad institucional. Oviedo: KRK.

Ostrom, E. y Walker, J. (2003). Trust and reciprocity. Interdisciplinary lessons from experimental research. New York: Russell Sage.

Patel, M., KoK, K. y Rothman, D. S. (2007). Participatory scenario construction in land use analysis: An insight into the experiences created 
by stakeholder involvement in the Northern Mediterranean. Land Use Policy, 24, 546-561. Peterson, G. D., Cumming, G. S. y Carpenter, S. R. (2003). Scenario planning: A tool for conservation in an uncertain world. Conservation Biology, $17,358-366$.

Raskin, P. G., Gallopin, P., Gutman, P., Hammond, A. y Swart, R. (1998). Bending the curve: Towards global sustainability. Stockholm: Environment Institute Stockholm.

Rincón-Ruiz, A. (2014). Can common property regimes in Colombia curb the expansion of coca crops and the deforestation? En Power, Justice and Citizenship: The Relationships of Power. McBain, D. (ed.). Oxford: Reino Unido, Interdisciplinary Press.

Rincón-Ruiz, A. y Kallis, G. (2013). Caught in the middle, Colombia's war on drugs and its effects on forest and people. Geoforum, 46, 60-78.

Rincón-Ruiz, A., Pascual, U. y Flantua, S. (2013). Examining spatially varying relationships between coca crops and associated factors in Colombia, using geographically weight regression. Applied Geography, 37, 23-33.

Rincón, S. A., Toro, J. y Burgos, J. (2009). Lineamientos guia para la evaluación de criterios de biodiversidad en los estudios ambientales requeridos para licenciamiento ambiental. Biodiversidad y estudios de impacto ambiental. Elementos para evaluadores. Bogotá: Instituto de Investigación de Recursos Biológicos Alexander von Humboldt e Instituto de Estudios Ambientales de la Universidad Nacional de Colombia.
Rhodes, R. (1997). Understanding Governance: Policy Networks, Governance, Reflexivity and Accountability. Buckingham: Open University Press.

Rodríguez-Becerra, M., Uribe, E. y Carrizosa, J. (1996). Instrumentos económicos para la gestión ambiental en Colombia. Santafé de Bogotá: Fescol, Cerec.

Rodríguez Becerra. M. y Espinoza, G. (2002). Gestión ambiental en América Latina y el Caribe: evolución, tendencias y principales prácticas (Wilk, D. ed.). Washington: Banco Interamericano de Desarrollo, Departamento de Desarrollo Sostenible, División de Medio Ambiente.

Rodríguez, K. J. y Ávila, V. (2014). Instrumentos de política pública para la conservación: su nacimiento y evolución en Colombia. Perfiles Latinoamericanos. 22 (43), 127-158.

Solano, C. (2001). Estudio de caso: estrategia de conservación en tierras privadas. En Jiménez, A., Solano, C., Barrera, M. P. y Barrera, M. X. (eds.). Las servidumbres ecológicas. Un mecanismo para la conservación en tierras privadas. Memorias taller, Bogotá, mayo 29 y 30 de 2001. Fundación Natura, Red de Reservas Naturales de la Sociedad Civil, The Nature Conservancy, www Colombia.

Subirats, J. (2010). Si la respuesta es gobernanza, ¿̨cuál es la pregunta? Factores de cambio en la política y en las políticas. Ekonomiaz, 2 (74), 16-35.

Tacconi, L. (2012). Redefining payments for environmental services. Ecological Economics, 73 (1), 29-36.

Wunder, S. (2006). Payments for environmental services: Some nuts and bolts. Bogor, Indonesia: Centro Internacional de Investigación Forestal (Cifor). 